\title{
Comparative study of chitosan and chitosan-gelatin scaffold for tissue engineering
}

\author{
Pawan Kumar $^{1} \cdot$ Brijnandan S. Dehiya $^{1} \cdot$ Anil Sindhu $^{2}$
}

Received: 26 September 2016 / Accepted: 15 November 2017 / Published online: 6 December 2017

(C) The Author(s) 2017. This article is an open access publication

\begin{abstract}
A number of orthopedic disorders and bone defect issues are solved by scaffold-based therapy in tissue engineering. The biocompatibility of chitosan (polysaccharide) and its similarity with glycosaminoglycan makes it a bone-grafting material. The current work focus on the synthesis of chitosan and chitosan-gelatin scaffold for hard tissue engineering. The chitosan and chitosan-gelatin scaffold have shown improved specific surface area, density, porosity, mechanical properties, biodegradability and absorption. These scaffolds can lead to the development or artificial fabrication of hard tissue alternates. The porous scaffold samples were prepared by freeze-drying method. The microstructure, mechanical and degradable properties of chitosan and chitosan-gelatin scaffolds were analyzed and results revealed that the scaffolds prepared from chitosan-gelatin can be utilized as a useful matrix for tissue engineering.
\end{abstract}

Keywords Chitosan $\cdot$ Gelatin $\cdot$ Scaffold $\cdot$ Glycosaminoglycan $\cdot$ Freeze drying $\cdot$ SEM

\section{Introduction}

Chitosan (natural polysaccharide) is a derivative of chitin [poly ( $N$-acetyl-1, 4- $\beta$-D-glucopyranosamine)], used in tissue regeneration due to its biocompatibility, biodegradation, antimicrobial, and immunogenic properties. Gelatin is derived from naturally occurring collagen by hydrolysis. It is an appropriate biomaterial because its cost and immunogenicity is low. Gelatin has good biodegradability, compatibility with living tissue, cell adhesion and proliferation properties. Gelatin is amalgamated with chitosan to enhance its biological activity. The presence of Arg-Gly-Asp (RGD)like sequence in gelatin stimulates cell adhesion, immigration and form a polyelectrolyte complex [1]. Bone tissue defects and diseases are increasing due to the consequence of trauma, injury, infections, besides this degenerative bone loss are of major concern in the field of human health. Every

Pawan Kumar

pawankamiya@yahoo.in

1 Department of Materials Science and Nanotechnology, Deenbandhu Chhotu Ram University of Science and Technology, Sonipat 131039, India

2 Department of Biotechnology, Deenbandhu Chhotu Ram University of Science and Technology, Sonipat 131039, Haryana, India year, a frightening increase in the number of bone injuries is estimated to continue around the globe, which leads to high demand for bone substitutes [2]. Failure rates in bone regeneration techniques are attributed to several reasons same as disease transfer, immune system rejection and donor crisis $[3,4]$. Tissue engineering has the potential to produce tissues and organs de novo. The required pore sizes for bone, muscle and skin are 100-300 $\mu \mathrm{m}$ [5], 100-200 $\mu \mathrm{m}[6,7]$, and $20-120 \mu \mathrm{m}$ [8], respectively. The tissue engineering materials for scaffold should be biocompatible, biodegradable, and mechanically stable, which depends on the material's chemistry, solubility, structure, shape, hydrophobicity/ hydrophilicity, surface energy, water absorption, molecular weight. Beyond these cell proliferation, migration and differentiation to make specific tissue while secreting the extracellular matrix components required to make the tissue. In tissue engineering, temporary scaffold enhances cell adhesion, maintenance of different functions without blocking proliferation. Scaffolds imitate the extracellular matrix and have a crucial role to play in supporting cell growth, differentiation and in delivering growth factors or other bioactive molecules [9]. The scaffolds are applied as a biological and physical back up for cell formation and for transplanting them into an organism. The final products of scaffold degradation must not be toxic and be capable of being removed from the body without conflict with other 
organs. Some materials have appropriate mechanical properties, but due to high porosity they failed when implanted in vivo due to insufficient capacity for vascularization. The progress of any scaffold is the equity between mechanical properties and porous structure which allow cell intrusion and vascularization. The cationic nature of chitosan scaffold exhibit electrostatic interactions with negatively charged molecules such as anionic glycosaminoglycan (GAG), proteoglycans and others [10]. Glycosaminoglycan (GAG) is a core component of extracellular matrix in tissues that builds up cell adhesion, proliferation and cell function. In many studies, chitosan can be easily molded into different forms such as porous 3D structures [11], gels [11], thin films [12], membranes [13] and fibers [14] which are favorable for bone cell growth. However, chitosan lacks sufficient mechanical strength, cell binding ability, rapid degradation and lacking of bioactive cell signaling molecules which are vital for bone tissue regeneration [15]. To overcome this problem, gelatin was used with the chitosan in this work.

\section{Materials and methods}

\section{Materials used}

Chitosan was purchased from HIMEDIA laboratories (P) Ltd. (Mumbai, India). Glutaraldehyde was purchased from TCI Chemicals (P) Ltd. (Chennai, INDIA). Sodium hydroxide pellets AR, acetic acid were purchased from SISCO Research Laboratories (P) Ltd. (Mumbai, India). Tri pyrophosphate was purchased from LobaChemie (P) Ltd. (Mumbai, India). Lysozyme was taken from SRL. Gelatin Powder Bacto was taken from S. D. fine chemicals Ltd. (Mumbai, INDIA). Sodium borohydride was purchased from SigmaAldrich Pvt, Ltd. New Delhi.

\section{Scaffold Synthesis}

The freeze-drying method was used to synthesize chitosan and chitosan-gelatin scaffolds. A $1 \%(\mathrm{w} / \mathrm{v})$ acetic acid was added into $2 \%(\mathrm{w} / \mathrm{v})$ solution of chitosan to make chitosan scaffold. To make chitosan-gelatin scaffold, $4 \%$ (w/v) gelatin solution was added into the solution under agitation for $12 \mathrm{~h}$ at $37{ }^{\circ} \mathrm{C}$. To make cross-linking, glutaraldehyde $0.25 \%$ was added to the mixture solution. Then mixture solution was dispensed into Petri dishes $\left(75 \mathrm{~mm}^{2}\right)$. These dishes placed within an ultra-low temperature freezer (LFZ-86L series, LABFREEZ) to freeze the samples at $-80{ }^{\circ} \mathrm{C}$ temperatures for $12 \mathrm{~h}$. After that the frozen samples were lyophilized until dry in a freeze dryer (ALLIED FROST, Macflow Engg. Pvt. Ltd.) for $24 \mathrm{~h}$ where the ice is removed by direct sublimation. The dried (lyophilized) scaffold samples were neutralized by $10 \% \mathrm{NaOH}$ to remove acetate followed by distilled water washing. To block non-responded aldehyde groups, these scaffolds were washed with double distilled water and sodium borohydride solution $(5 \%)$ before repeating the freeze-drying process [16].

\section{Characterizations}

To investigate the structure and properties following characterization methods has been used.

\section{Scanning electron microscopy}

The lyophilized sample scaffolds were characterized for their surface and fracture sections observation. The sample scaffolds were coated with Au prior and examined by scanning electron microscope (JEOL). All chitosan platforms were smooth, delicate, sponge-like, adaptable and sufficiently solid to handle in wet and dry conditions without twisting. The powerful size of the pore was figured as the mean distances across of scaffold pores. No less than 12 pores were surveyed from three unique areas of a similar example. The values were expressed as the mean \pm standard error.

\section{Surface area and porosity}

BELSORP mini (MicrotracBEL Corp.) was used to measure specific surface area, pore specific surface area, pore volume and micropore radius of scaffold samples. It works on the basis of volumetric gas adsorption method + AFSM. During the measurement, it is essential to maintain the liquid coolant level to keep free space in the sample cell which is practically difficult to achieve. Sample cell, frit and cap are dried and weighed before adding the sample in the tube (cell weight). Sample is cut into small pieces and inserted in the sample tube; the sample is loaded in the BELPREP vac II and degassed at $105^{\circ} \mathrm{C}$ for $2 \mathrm{~h}$ at a vacuum pressure of $10-2 \mathrm{kPa}$ (cell + sample weight). The sample cell is then removed and weighed. The difference in weight gives the sample quantity. The sample cell is loaded in the BELSORP mini, sample table and measurement condition (Pressure $133 \mathrm{kPa}$ and holding time $30 \mathrm{~h}$ ) is entered in the software and analysis is started. The observed results are shown in the Table 1. The micropore radii largely depend on concentration and viscosity of the solution. Additionally, these properties depend on the $\mathrm{pH}$, temperature and the rate of freeze drying.

\section{Measurement of density}

The density of sample scaffold was computed from the proportion of the weight of the freeze dried scaffold to their 
Table 1 Porosity and density of chitosan and chitosan-gelatin scaffold

\begin{tabular}{llllll}
\hline S. no. & Sample name & $\begin{array}{l}\text { Specific surface } \\
\text { area }\left(\mathrm{m}^{2} \mathrm{~g}^{-1}\right)\end{array}$ & $\begin{array}{l}\text { Pore-specific surface } \\
\text { area }\left(\mathrm{m}^{2} \mathrm{~g}^{-1}\right)\end{array}$ & $\begin{array}{l}\text { Pore volume } \\
\left(\mathrm{cm}^{3} \mathrm{~g}^{-1}\right)\end{array}$ & $\begin{array}{l}\text { Micropore } \\
\text { diameter } \\
(\mathrm{nm})\end{array}$ \\
\hline 1. & Chitosan & 1.9185 & 2.0965 & 0.0026 & 2.44 \\
2. & Chitosan + gelatin & 1.9832 & 2.0384 & 0.0027 & 2.44 \\
\hline
\end{tabular}

volume. The density of chitosan and chitosan-gelatin scaffolds was calculated by the formula [16],

$\rho=\frac{W}{\pi \times(D / 2)^{2} \times H}$,

where $\rho$ is the density, $D$ is the diameter, $H$ is the thickness, and $W$ the weight of the scaffold, respectively. The values are reported as the mean \pm standard error.

\section{PBS solution adsorption}

PBS solution adsorption showed the swelling capacity of sample scaffolds. It depends on porosity and the amount adsorbed is directly proportional to the porosity of the material in addition to its material properties such as hydrophilicity. The scaffolds were placed in a phosphate-buffered saline (PBS) at $7.4 \mathrm{pH}$ for 30 and $60 \mathrm{~min}$ at room temperature. The percentage adsorption capacities of the scaffolds were determined by the given formula [16],

Water adsorption $\%=\frac{W_{\mathrm{w}}-W_{\mathrm{d}}}{W_{\mathrm{d}}} \times 100$,

where $W_{\mathrm{w}}$ is final weight of the scaffolds after adsorption, and $W_{\mathrm{d}}$ is initial weight of the dry scaffolds.

The wet weight of the sample scaffold was calculated by first blotching the sample surface with filler paper to remove adsorbed water, then weighing quickly.

\section{In vitro degradation}

The weight loss observation was used to study the degradation of various scaffolds by calculating the change in scaffold samples weight. The samples of scaffolds were incubated in the solution of PBS at pH 7.4 with $1 \times 10^{4} \mathrm{U} / \mathrm{ml}$ of lysozyme concentration in incubation dish and kept at $37^{\circ} \mathrm{C}$ for 15 days. The particular concentration of lysozyme was related to the fixation in human serum. The solution for degradation was changed every day. After 15 days, the samples were put out from PBS solution, rinsed three times with distilled water and freeze dried for $24 \mathrm{~h}$. Then the dried samples were weighed and the change in weight was recorded in percentage. The degradation testing of the scaffolds was calculated by the given formula [16],

Weight degraded $(\%)=\frac{W_{\mathrm{f}}-W_{0}}{W_{0}} \times 100$,

where $W_{\mathrm{f}}$ is the final weight of scaffold sample after degradation and $W_{0}$ is the initial weight of scaffold sample.

\section{Mechanical property measurement}

The fracture strength of the sample scaffolds was measured. The specimen of $2.5 \times 4 \times 4 \mathrm{~mm}^{3}$ sizes was used for testing. The values were expressed as the mean \pm standard error. Lyophilized scaffolds at predetermined time points were used for mechanical testing. The force bearing capacity of the scaffolds was expressed by a force vs time graph. The force-time graphs showing the fracture strength of the scaffolds are depicted in the Fig. 1.

\section{Bulk structure}

Both chitosan and chitosan-gelatin samples were examined by fluorescence microscope (Leica DM 250). The interconnected heterogeneous chain structures and pore arrangements in both samples were examined. The difference in pore arrangements and chain structures between chitosan and chitosan-gelatin scaffolds has measured.

\section{Results and discussion}

The morphology of this specimen significantly displays open pore structure with both micropores and macropores. An open pore structure is good for blood supply and cell attachment. The heterogeneous pore microstructures in all the chitosan scaffolds and well interconnected pores are shown in Fig.2. Stretched pores were also observed in the sample scaffolds whose formation might be due to growth highly parallel ice crystals in the sample scaffold substrate and the creation of hydrogen bonds in the long chains of polymers. In another work [7, 16], the observed pore sizes were $50-100 \mu \mathrm{m}$ and $102 \pm 27 \mu \mathrm{m}$ in scaffold while the porosity of scaffold synthesis in present work is 9.3-36.2 $\mu \mathrm{m}$ and 23.2-189.4 $\mu \mathrm{m}$. So chitosan-gelatin scaffolds can be more 


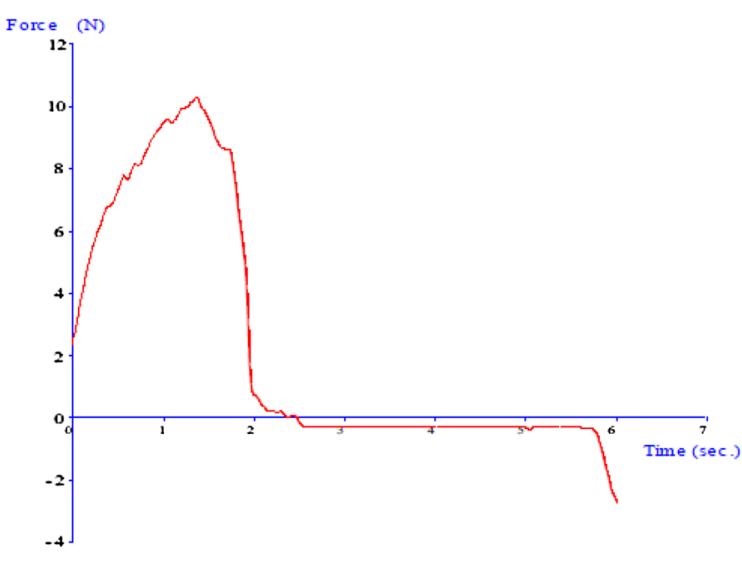

(a)

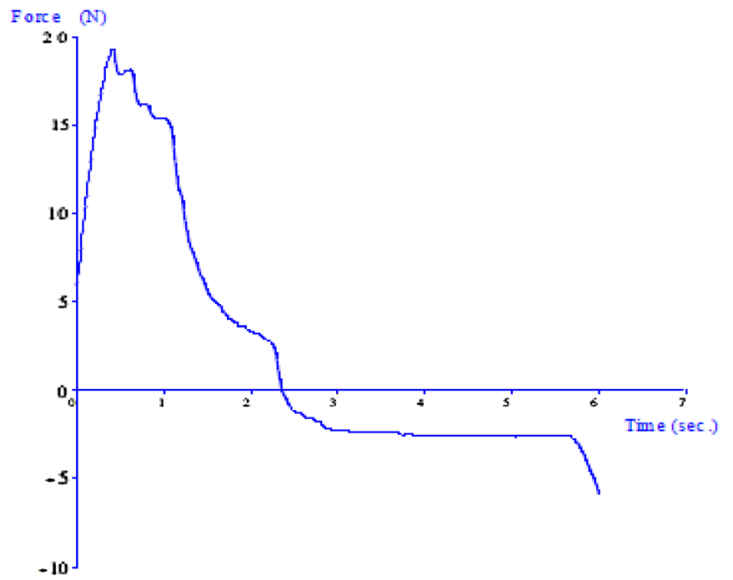

(b)

Fig. 1 Fracture strength graph of chitosan (a) and chitosan-gelatin (b) scaffolds

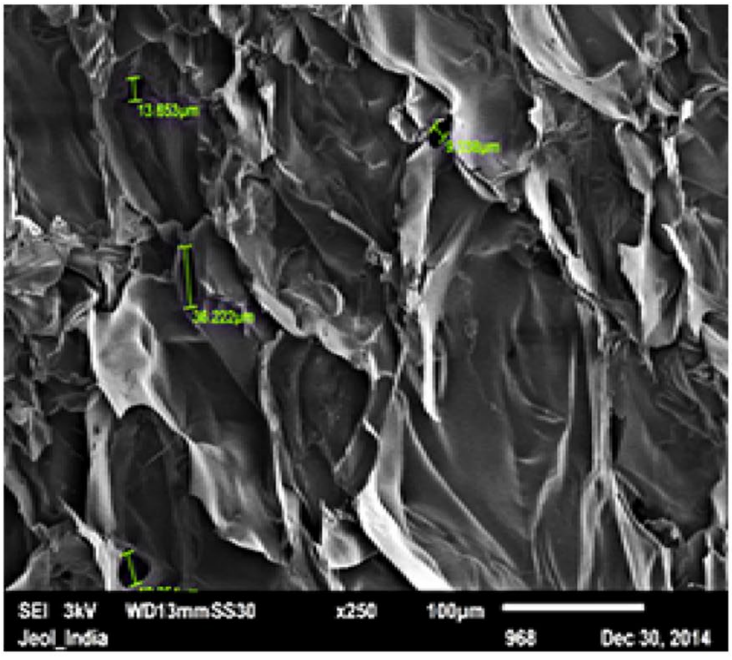

(a)

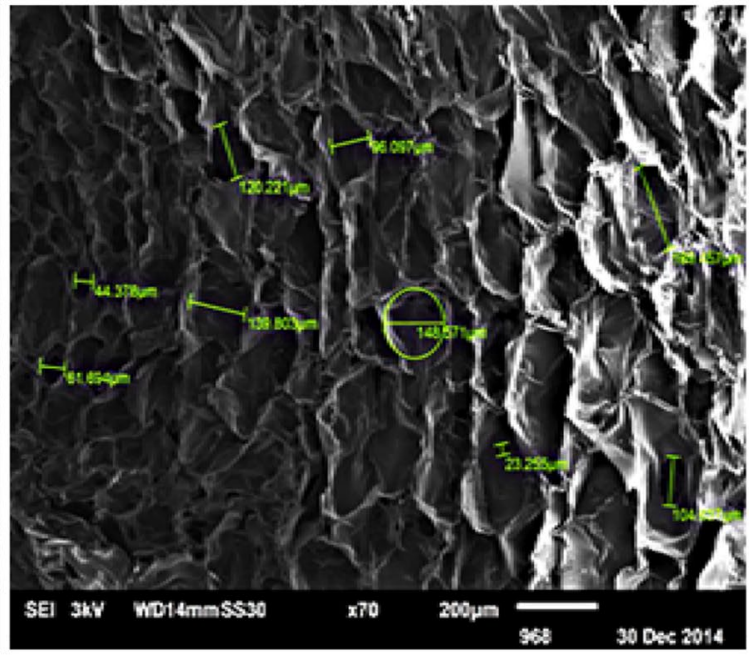

(b)

Fig. 2 SEM image of chitosan (a) scaffold and chitosan-gelatin (b) scaffold

suitable for tissue engineering than metallic scaffold. The average pore size below $300 \mu \mathrm{m}$ helps in osteoblast and cell proliferation in scaffold. The small pore sizes display osteoblast survival and bone formation. Figure 3 revealed the bulk structure and distribution of chitosan and gelatin phases in the scaffolds. The pores are enlarged, heterogeneous and perpendicular in the chitosan-gelatin sample scaffold while the pores were loosely packed and small sized in chitosan sample scaffold. The pore sizes in the chitosan-gelation sample scaffold are influenced by gelation concentration. Some hair-like structures were found in chitosan-gelatin sample. The sample of chitosan-gelatin showed low density as compare to chitosan sample. Pre-freezing temperature is another parameter, which has effect on the mean pore sizes in addition to gelatin composition concentration. Due to addition of gelatin, the pore sizes get to be distinctly smaller and pore dividers more slender, while interconnectivity along declining pre-solidifying temperature. The specific surface area of chitosan-gelatin scaffold was formed to be $1.9832 \mathrm{~m}^{2} / \mathrm{g}$ and chitosan scaffold was $1.9185 \mathrm{~m}^{2} / \mathrm{g}$. The measured density of the scaffold is inversely proportional to the volume of the sample. The densities of chitosan and chitosan-gelatin scaffold were found to be 0.06071 and $0.05350 \mathrm{~g} / \mathrm{cm}^{3}$, respectively. The observed water adsorption capacity of chitosan and chitosan-gelatin scaffolds after 30 min was found to be 35 and $38 \%$ by weight. After $60 \mathrm{~min}$ the water adsorbed by 


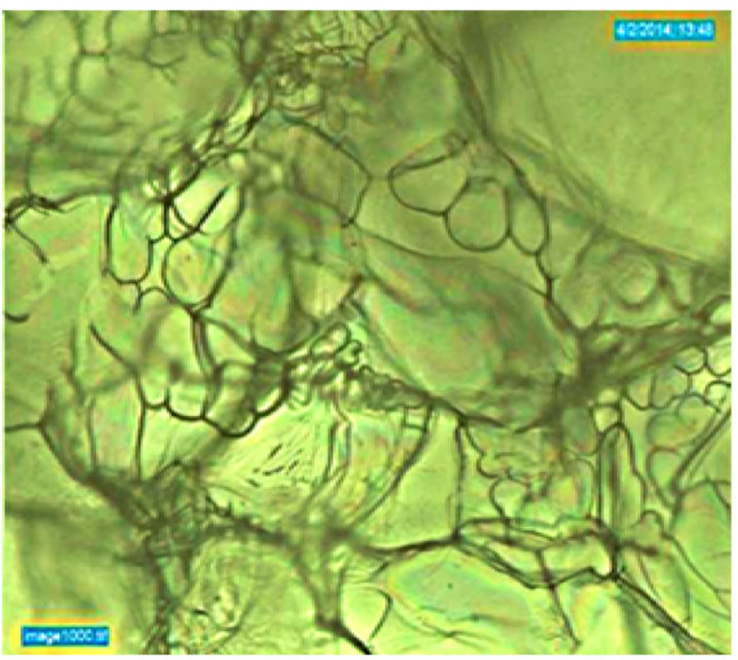

(a)

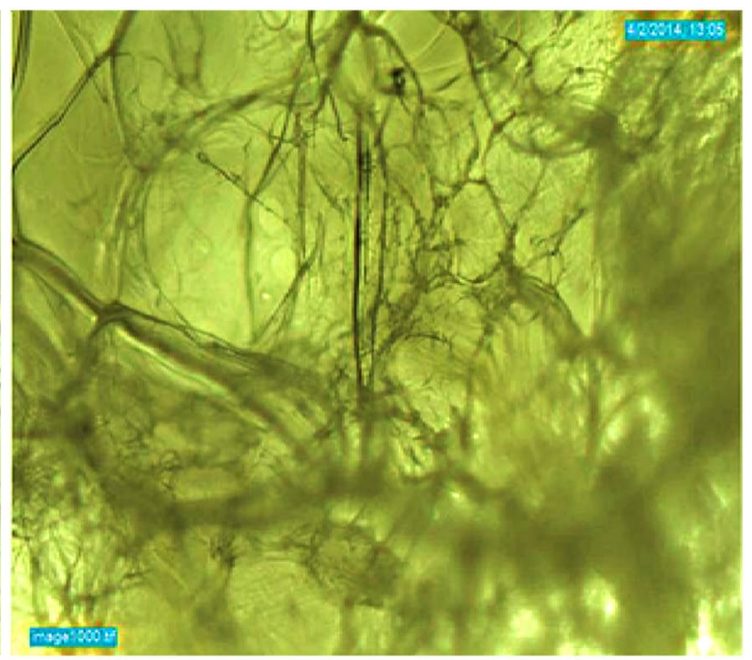

(b)

Fig. 3 Microscopic image of chitosan (a) and chitosan-gelatin (b) scaffold

chitosan and chitosan-gelatin scaffolds was 41 and $46 \%$. The initial adsorption was found very fast in first $30 \mathrm{~min}$. The water adsorption capacity of chitosan scaffold was 3-5\% higher than chitosan-gelatin scaffold. A very slow adsorption percentage increase was also noticed after $60 \mathrm{~min}$. The results reveal that the pre-freezing temperature had some effect on the deterioration of the scaffold by influencing the structure of the scaffold. The lower pre-freezing temperature, leading to smaller pore wall, would promote scaffold degradation. The percentage degradation of chitosan and chitosan-gelatin scaffolds was $47.68 \%$ and $79.40 \%$ by weight separately. The in vitro degradation of chitosan-gelatin scaffold is $31.72 \%$ higher than chitosan scaffold. Therefore, biodegradation is probably occurring along with water ion usually in volatile phases. The degradability of scaffolds depends upon the degree of crystallinity, which controls the hydrolysis rate. The graph indicates that the force-bearing capacity of chitosan scaffold was $1047.6 \mathrm{~N} / \mathrm{m}^{2}$ (equivalent stress of $1.05 \mathrm{MPa}$ ) and breaking time was $1.38 \mathrm{sec}$ after application of force on the sample. In Fig. 3b, the forcebearing capacity of chitosan-gelatin scaffold was found to be $1966.2 \mathrm{~N} / \mathrm{m}^{2}$ (equivalent stress of $1.9 \mathrm{MPa}$ ) with breaking time of $0.432 \mathrm{~s}$. (Loading rate was $3 \mathrm{~cm} / \mathrm{min}$ ).

\section{Conclusion}

The prepared chitosan and chitosan-gelatin sample scaffolds were colorless and inelastic. In these scaffolds, the chitosan-gelatin scaffold revealed the comparative properties of high porosity, optimum pore size distribution and low density with good tensile mechanical properties. The combination of gelatin with chitosan improved the mechanical properties and biodegradation kinetics of scaffold, formed a prominent amorphous composite. The chitosan-gelatin scaffold showed uniformities in porosity. The higher tensile strength was observed in scaffolds prepared by chitosan-gelatin due to the possible formation of durable hydrogen bonds during preparation of samples. The water adsorption capacity of chitosan-gelatin sample was also higher. In conclusion, this work highlights chitosan-gelatin scaffolds can be used as an improved chitosan scaffold material for tissue engineering.

Open Access This article is distributed under the terms of the Creative Commons Attribution 4.0 International License (http://creativecommons.org/licenses/by/4.0/), which permits unrestricted use, distribution, and reproduction in any medium, provided you give appropriate credit to the original author(s) and the source, provide a link to the Creative Commons license, and indicate if changes were made.

\section{References}

1. Thein-Han, W.W., Saikhun, J., Pholpramoo, C., Misra, R.D.K., Kitiyanant, Y.: Chitosan-gelatin scaffolds for tissue engineering: physico-chemical properties and biological response of buffalo embryonic stem cells and transfectant of gfp-buffalo embryonic stem cells. Acta. Biomater. 5(9), 3453-3466 (2009)

2. Costa-Pinto, Ana Rita, Reis, Rui L., Neves, Nuno M.: Scaffolds based bone tissue engineering: the role of chitosan. Tissue Eng. Part B 17(5), 331-347 (2011)

3. Mohammadi, Y., Mirzadeh, H., Moztarzadeh, F., Soleimani, M., Jabbari, E.: Osteogenic differentiation of mesenchymal stem cells on novel three-dimensional poly(L-lactic acid)/chitosan/gelatin/ $\beta$-tricalcium phosphate hybrid scaffolds. Iran. Polym. J. 16(1), 57-69 (2007)

4. Sabir, Muhammad Iqbal, Xiaoxue, Xu, Li, Li: A review on biodegradable polymeric materials for bone tissue engineering applications. J. Mater. Sci. 44(21), 5713-5724 (2009) 
5. Hutmacher, Dietmar W.: Scaffold design and fabrication technologies for engineering tissues - state of the art and future perspectives. J. Biomater. Sci. Polym. Ed. 12(1), 107-124 (2001)

6. Beier, Justus P., et al.: Collagen matrices from sponge to nano: new perspectives for tissue engineering of skeletal muscle. BMC Biotechnol. 9, 34 (2009)

7. Danielsson, Carina, Ruault, Sylvie, Simonet, Marc, Neuenschwander, Peter, Frey, Peter: Polyesterurethane foam scaffold for smooth muscle cell tissue engineering. Biomaterials 27(8), 1410-1415 (2006)

8. Kim, S.S., et al.: Survival and function of hepatocytes on a novel three-dimensional synthetic biodegradable polymer scaffold with an intrinsic network of channels. Ann. Surg. 228(1), 8-13 (1998)

9. Martins, Ana M., Alves, Catarina M., Kurtis Kasper, F., Mikos, Antonios G., Reis, Rui L.: Responsive and in situ-forming chitosan scaffolds for bone tissue engineering applications: an overview of the last decade. J. Mater. Chem. 20(9), 1638-1645 (2010)

10. Martino, Di, Alberto, Michael Sittinger, Risbud, Makarand V.: Chitosan: a versatile biopolymer for orthopaedic tissue-engineering. Biomaterials 26(30), 5983-5990 (2005)

11 Yamamoto, Masaya, Takahashi, Yoshitake, Tabata, Yasuhiko: Controlled release by biodegradable hydrogels enhances the ectopic bone formation of bone morphogenetic protein. Biomaterials 24(24), 4375-4383 (2003)
12 Naznin Sultana and Mohammed Rafiq Abdul Kadir: Study of in vitro degradation of biodegradable polymer based thin films and tissue engineering scaffolds. Afr. J. Biotech. 10(81), 1870918715 (2011)

13 Orrego, Carlos E., Valencia, Jesus S.: Preparation and characterization of chitosan membranes by using a combined freeze gelation and mild crosslinking method. Bioprocess Biosyst. Eng. 32(2), 197-206 (2009)

14 Pati, Falguni, Kalita, Hemjyoti, Adhikari, Basudam, Dhara, Santanu: Osteoblastic cellular responses on ionically crosslinked chitosan-tripolyphosphate fibrous 3-D mesh scaffolds. J. Biomed. Mater. Res. Part A 101A(9), 2526-2537 (2013)

15 Porter, Joshua R., Ruckh, Timothy T., Popat, Ketul C.: Bone tissue engineering: a review in bone biomimetics and drug delivery strategies. Biotechnol. Prog. 25(6), 1539-1560 (2009)

16 Mao, Jin Shu, Zhao, Li Guo, Yin, Yu Ji, De Yao, Kang: Structure and properties of bilayer chitosan-gelatin scaffolds. Biomaterials 24(6), 1067-1074 (2003)

Publisher's Note Springer Nature remains neutral with regard to jurisdictional claims in published maps and institutional affiliations. 